\title{
Nonalcoholic Fatty Liver Disease in Patients with Autoimmune Hepatitis: Further Reason for Teeth GNASHing?
}

\author{
Christina Weiler-Normann ${ }^{1} \cdot$ Ansgar W. Lohse $^{1}$
}

Published online: 28 July 2016

(c) Springer Science+Business Media New York 2016

\begin{abstract}
Abbreviations
NASH Nonalcoholic steatohepatitis

NAFLD Nonalcoholic fatty liver disease

AIH Autoimmune hepatitis

RR Relative risk
\end{abstract}

Nonalcoholic steatohepatitis (NASH), the aggressive form of nonalcoholic fatty liver disease (NAFLD), is a public health burden of increasing significance associated with significant liver-related and -unrelated morbidity and mortality [1]. Predisposing factors, apart from a partially defined genetic predisposition, include the presence of diabetes or pre-diabetes, hyperlipidemia, obesity, and lack of physical activity and exercise. This emerging disease, largely unknown only few decades ago, has developed into a worldwide health epidemic affecting not only 10-30\% of the Western population, but also increasing portions of populations in other parts of the world, including developing countries.

In view of the high and increasing prevalence rates, NASH is likely to be superimposed on other chronic liver diseases. Statistically, 10-30 \% of any particular liver disease population should be affected by NAFLD, with a lesser percentage affected by NASH. This coincidence presents a challenge for physicians, both in making the correct diagnoses as well as in managing these coincident and possibly comorbid diseases, despite the medical school

Ansgar W. Lohse

alohse@uke.de

1 Department of Medicine, University Medical Center Hamburg-Eppendorf, Martinistrasse 52, 20246 Hamburg, Germany dictum that a single diagnosis should be sought for a complaint or for abnormal laboratory values. This paradigm is clearly outdated when considering liver diseases, as beautifully illustrated in the publication by De LucaJohnson et al. [2] in this issue of Digestive Diseases and Sciences. This straightforward study analyzing the liver histology of a consecutive group of 73 patients with a diagnosis of autoimmune hepatitis (AIH) at a single center reported that $12 / 73(16 \%)$ of the studied patients showed histologic features of both NASH and $\mathrm{AIH}$, with a further $10 / 73(14 \%)$ simple steatosis. This prevalence rate is within the expected rate in the general population and therefore unsurprising. Yet, these data may underestimate the true prevalence since some patients with coincident $\mathrm{NASH}$ and AIH were likely originally misdiagnosed as only suffering from NASH and thus did not qualify for this study. Be that as it may, since this study very clearly demonstrates that the two conditions can and do coexist in a substantial number of patients, clinicians and pathologists caring for patients with liver disease need to be aware of this possibility. Clearly, AIH no longer is a disease "of exclusion," but has to be diagnosed on the basis of history, laboratory findings, serology, and biopsy findings [3], with a trial of immunosuppressive therapy sometimes needed to support the diagnosis [4].

Are NASH and AIH purely coincident in these patients or do these conditions influence each other? Although it is difficult to answer this question on basis of the available evidence, this study provides at least some clues: $50 \%$ of the AIH/NASH patients, but only $18 \%$ of the AIH patients without concomitant fatty liver disease were cirrhotic according to the initial index biopsy. This difference suggests that patients with AIH/NASH run a more aggressive disease course than do patients with AIH alone, an observation that could be supported by data obtained from 
Fig. 1 Treatment algorithm for patients with AIH/NASH.

Patients with features of NASH should receive adequate testing for metabolic syndrome (HbA1c, glucose tolerance, arterial hypertension, hyperlipidemia) in addition to counseling regarding lifestyle adjustments

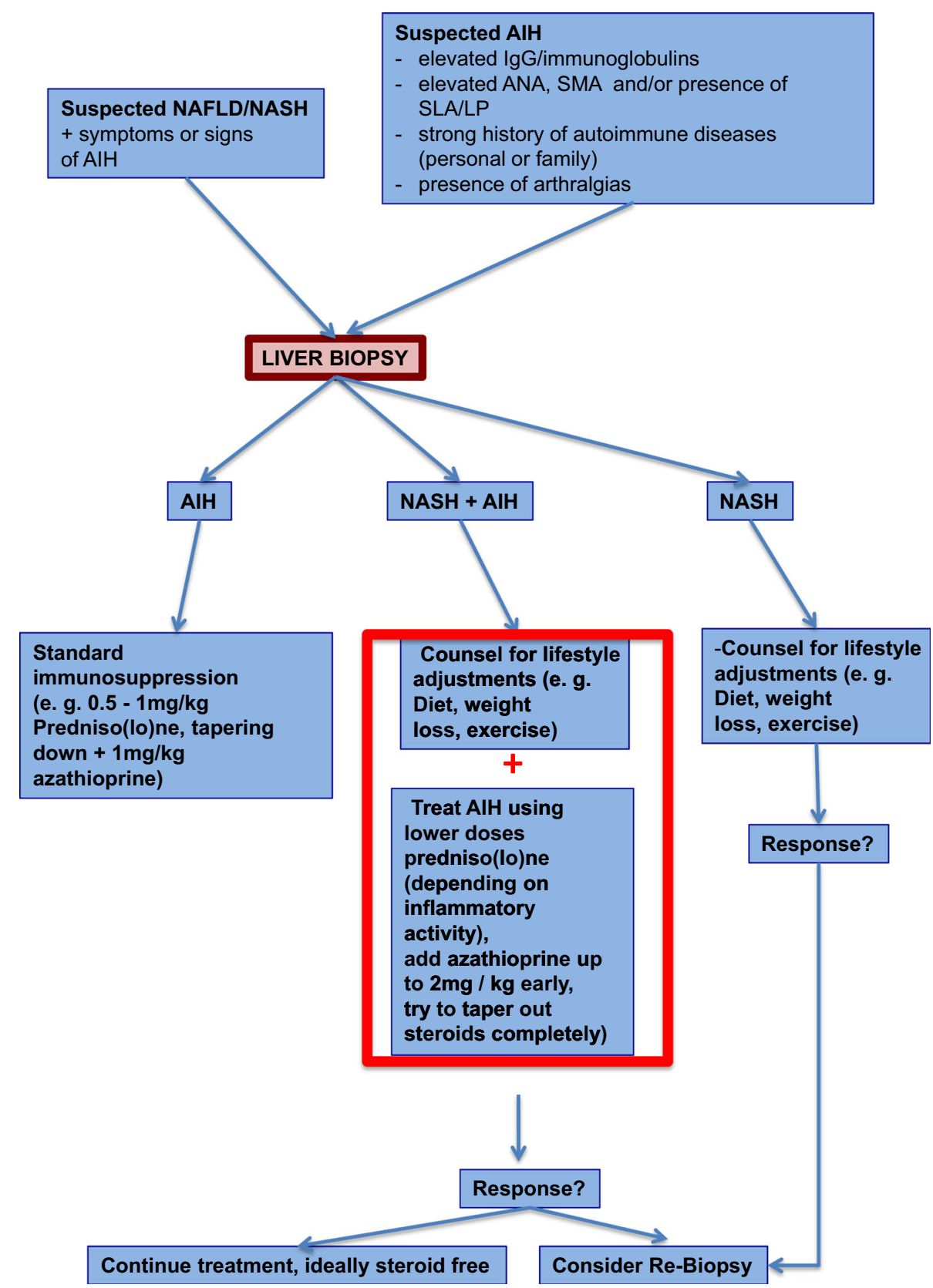

patients with NASH and other liver diseases such as hepatitis C [5]. Pathophysiologically, one explanation for this finding could be that the inflammatory infiltrate within the liver is largely made up of bystander immune cells with only few actual antigen-specific lymphocytes. These bystander cells could react nonspecifically to a second damaging agent and thereby aggravate the inflammation and subsequent liver damage, leading to a more aggressive disease course.

Nevertheless, there is an important alternative explanation: Patients with NASH and AIH were diagnosed later in the disease course than patients with AIH alone. The mean age of the AIH alone group was 48 , compared with 58 in the AIH/NASH patients, a difference that just missed statistical significance $(p=0.077)$, likely due to the relatively small number of cases. Nonetheless, diagnostic delay may be the main problem in these patients. Since AIH/NASH patients displayed the typical risk profile of diabetes, obesity, and hyperlipidemia, it is likely that in many of these patients, elevated liver enzymes were interpreted by primary caregivers as an expression of NASH without considering alternative or additional diagnoses. A similar phenomenon of delayed diagnosis has been described particularly in elderly men with $\mathrm{AIH}$, in whom the 
diagnosis appears to be significantly delayed [6]. The present study strongly supports the importance of a liver biopsy and its careful expert evaluation, even if the diagnosis of AIH or of NASH seems to be obvious on the basis of the laboratory findings (Fig. 1).

The second major finding of the paper by the De LucaJohnson group is the difference in outcomes: AIH/NASH patients had a markedly increased relative risk (RR) for adverse liver-related outcomes (RR 2.55) and for liverrelated mortality (RR 7.65), a mortality risk that remained significant even when the groups were age-adjusted by excluding the younger patients from the AIH group, raising the average age to 55 (RR 6.49). This result, though disturbing and challenging, is yet not entirely surprising since both conditions have an increased risk in and of themselves. Nonetheless, the question is whether the combined risk is additive or synergistic. The data suggest that the risk may be disproportionately high: More aggressive inflammation may be due to the above-discussed bystander effects in addition to treatment-related effects such as those due to frequently prescribed corticosteroid therapy, which may have a detrimental effect on the course of NASH. Furthermore, the prognosis of AIH is decisively influenced by the effectiveness of the immunosuppressive therapy; patients not achieving a full remission have an increased risk of progressive cirrhosis and liver-related complications, whereas patients reaching a full remission with absent or minimal residual hepatic inflammatory activity have an excellent prognosis. It is likely that patients with AIH/NASH were more difficult to manage and may not have received the necessary dose of immunosuppression to achieve stable long-term remission, a supposition unsupported by the lack of reported significant management differences. Still, in view of the complexity of individualized management of AIH and in view of the relatively small patient numbers, this effect may have been too difficult to detect statistically. Much of the difference, or perhaps even all of the difference, may also have been due to the diagnostic delay and the pre-existing cirrhosis in half the AIH/NASH patients.

Standard treatment of AIH is comprised of induction therapy (usually with prednisone or prednisolone) and maintenance treatment with a steroid-sparing agent, mostly azathioprine or 6-mercaptopurine [4, 7]. Induction treatment with high-dose steroids will worsen diabetes and degrade the metabolic state of the patients by causing additional weight gain. A treatment protocol with lower initial steroid doses and most importantly a rapidly tapering steroid dose using alternative immunosuppressants as early as possible is probably warranted. In our experience, shortterm steroids even at high doses are not detrimental to $\mathrm{AIH} /$ NASH patients, but glucose homeostasis may be a problem. Long-term steroid treatment (beyond 6-month induction therapy), however, can be quite harmful. It thus remains to be shown if initial therapy, by inducing rapid remission with high-dose steroids, will enable overall steroid sparing and thus be appropriate even for $\mathrm{AIH} /$ NASH patients.

In managing AIH/NASH patients, it is important to always consider both diseases and not just focus on immunosuppressive schedules: counseling of these patients (dietary advice, closer blood sugar surveillance, adaptation of diabetes medication, avoidance of weight gain, more physical exercise) is essential and should be repeated as appropriate. Dr. De Luca-Johnson and colleagues have highlighted an ever-emerging important clinical and scientific problem that warrants more future research both into the pathogenic factors and also into more appropriate and effective treatment approaches for these comorbid patients at high risk of liver-related morbidity and mortality.

\section{References}

1. European Association for the Study of the Liver. Clinical practice guidelines for the management of nonalcoholic fatty liver disease. J Hepatol. 2016;64:1388-1402.

2. De Luca-Johnson J, Wangensteen KJ, Hanson J, Krawitt EL, Wilcox R. Natural history of patients presenting with autoimmune hepatitis and coincident nonalcoholic fatty liver disease. Dig Dis Sci. (Epub ahead of print). doi:10.1007/s10620-016-4213-3.

3. Hennes EM, Zeniya M, Czaja AJ, et al. Simplified criteria for the diagnosis of autoimmune hepatitis. Hepatology. 2008;48:169-176.

4. European Association for the Study of the Liver. Clinical practice guidelines: autoimmune hepatitis. J Hepatol. 2015;63:971-1004.

5. Dyal HK, Aguilar M, Bhuket T, et al. Concurrent obesity, diabetes, and steatosis increase risk of advanced fibrosis among $\mathrm{HCV}$ patients: a systematic review. Dig Dis Sci. 2015;60:2813-2824.

6. Schramm C, Kanzler S, Zum Buschenfelde KH, Galle PR, Lohse AW. Autoimmune hepatitis in the elderly. Am J Gastroenterol. 2001;96:1587-1591.

7. Manns MP, Czaja AJ, Gorham JD, et al. Diagnosis and management of autoimmune hepatitis. Hepatology. 2010;51:2193-2213. 